\title{
偶氮和亚氨基团作为下垂螯合臂的新型杯[4]芳烃衍生物的合成及性质
}

\author{
范平*徐生明李巍巍陈辰祃艳强 \\ (辽宁大学化学院 沈阳 110036)
}

\begin{abstract}
摘要 以对叔丁基杯 [4]芳烃为原料, 经过取代、缩合等多步反应, 合成了 5 个下缘同时含有偶氮和亚氨基团的新型杯 [4]芳烃衍生物，其结构经 IR、 ${ }^{1} \mathrm{H} N \mathrm{~N}$ 、 ${ }^{13} \mathrm{C} N \mathrm{NMR}$ 和元素分析进行了表征. 并通过 UV/Vis 光谱研究了其对金属离子的 识别作用. 结果发现化合物 25,27-二差基-26,28-二\{2-[2-( $N$-(4-苯偶氮基-1-䒺基)亚氨基次甲基)苯氧基]乙氧基 $\}$ 对叔丁 基杯 [4] 芳烃(4c) 对 $\mathrm{Al}^{3+}$ 和 $\mathrm{Sn}^{2+}$ 有较好的识别作用, 而化合物 25,27-二羟基-26,28-二\{2-[2-( $N$-(4-(4-甲基苯偶氮基)-1-菜 基)亚氨基次甲基)苯氧基]乙氧基 $\}$ 对叔丁基杯 $[4]$ 芳烃 (4e)可以专一性地识别 $\mathrm{Al}^{3+}$, 其与 $\mathrm{Al}^{3+}$ 络合的物质的量比为 $1: 1$. 关键词＼cjkstart杯 [4]芳烃; 偶氮基团; 亚氨基团; UV/Vis 光谱
\end{abstract}

\section{Synthesis and Properties of Novel Azocalix[4]arene Derivatives with Azo and Imino Groups as the Lower Margin Chelating Arm}

\author{
Fan, Ping* Xu, Shengming Li, Weiwei Chen, Chen Ma, Yanqiang \\ (Institute of Chemistry, Liaoning University, Shenyang 110036)
}

\begin{abstract}
A serious of calix[4] arene derivatives containing azo and imino group in the lower rim were synthesized by substitution and condensation reactions from $p$-tert-butylcalix[4]arene. The structures of products were characterized by IR, ${ }^{1} \mathrm{H}$ $\mathrm{NMR},{ }^{13} \mathrm{C}$ NMR and elemental analysis. Their complexation behavior toward various metal ions was investigated by UV/vis

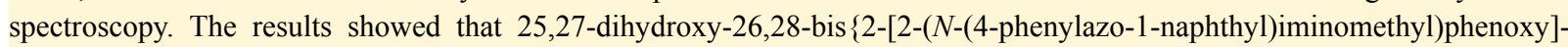
ethoxy\}-p-tert-butylcalix[4]arene (4c) could identify $\mathrm{Al}^{3+}$ and $\mathrm{Sn}^{2+}$, and 25,27-dihydroxy-26,28-bis $\{2-[2-(N-(4-(4-$ methylphenylazo)-1-naphthyl)iminomethyl)phenoxy]ethoxy\}-p-tert-butylcalix[4]arene (4e) could identify $\mathrm{Al}^{3+}$ specifically. The stoichiometry of $4 \mathrm{e}$ to $\mathrm{Al}^{3+}$ in the complex was $1: 1$.

Keywords calyx[4]arene; azo group; imino group; UV/Vis spectroscopy
\end{abstract}

杯芳烃被认为是继冠醚和环糊精之后出现的 “第三 代超分子”, 通过控制不同的反应条件, 可以改变杯芳 烃的空腔大小, 并可以对杯芳烃的上缘、下缘以及连接 苯环单元的亚甲基进行各种选择性官能化, 获得多种具 有高度选择性的主体分子 ${ }^{[1 ~ 6]}$. 许多含有亚氨基团(席夫 碱)的化合物具有抗真菌、抗肿瘤等生理活性, 并且其所 含结构单元 $\mathrm{C}=\mathrm{N}$ 可与多种离子形成稳定的配位化合 物 ${ }^{[7-9]}$. 而含有偶氮基团的化合物在染料、颜料及重金 属离子显色识别等领域得到广泛的应用, 连有偶氮基团 的杯芳烃衍生物在离子识别中可以达到肉眼识别的水 平 ${ }^{[10 ~ 14]}$. 将偶氮基团与亚氨基团结合到杯 [4]芳烃下缘 的同一支链, 形成下垂螯合臂, 利用其与杯芳烃空穴的
协同作用，可以增加所得杯芳烃衍生物对金属离子的选 择性络合能力, 并有可能根据反应中主体分子的色变监 测络合作用的发生.

天然水和动植物组织中存在的铝离子可以通过食 物链发生传递, 当其浓度过高时会杀死藻类和鱼 类 ${ }^{[15,16]}$. 现已有多项研究发现铝离子的富集会对人类的 中枢神经系统造成影响，导致多种疾病的发生 ${ }^{[17,18]}$. 因 而实现对铝离子的选择性识别具有重要意义.

本文以对叔丁基杯 [4]芳烃为原料, 经过取代、缩合 等多步反应, 合成了 5 个在同一支链上同时具有偶氮和 亚氨基团的新型杯 [4]芳烃衍生物 25,27-二羟基-26,28-二 \{2-[2-( $N$-(4-苯偶氮基苯基)亚氨基次甲基苯氧基]乙氧

*E-mail: pingfan_bft@126.com

Received December 17, 2014; revised February 6, 2015; published online March 3, 2015.

Project supported by the Liaoning Provincial Department of Education Fund for Scientific Research (No. L2011007) and the Shenyang Science and Technology Bureau of Science and Technology Program (No. F13-318-1-51).

辽宁省教育局科学研究基金(No. L2011007)和沈阳市科技局科技计划(No. F13-318-1-51)资助项目. 
基 $\}$ 对叔丁基杯 [4] 芳烃 (4a)，25,27-二羟基-26,28-二 \{2-[2-( $N$-(4-(2-甲基苯偶氮基)-2-甲基苯基)亚氨基次甲 基)苯氧基]乙氧基 $\}$ 对叔丁基杯 [4]芳烃(4b)，25,27-二羟 基-26,28-二 $\{2-[2-(N$-(4-苯偶氮基-1-菜基)亚氨基次甲基) 苯氧基]乙氧基 $\}$ 对叔丁基杯 [4]芳烃(4c), 25,27-二羟基26,28-二\{2-[2-( $N$-(4-(2-甲基苯偶氮基)-1-菜基)亚氨基次 甲基)苯氧基]乙氧基 $\}$ 对叔丁基杯 [4]芳烃(4d)和 25,27-二 羟基-26,28-二 $\{2-[2-(N-(4-(4-$ 甲基苯偶氮基)-1-菜基)亚 氨基次甲基)苯氧基]乙氧基 $\}$ 对叔丁基杯 [4]芳烃(4e) (Scheme 1). 目标化合物的结构通过 IR、 ${ }^{1} \mathrm{H}$ NMR、 ${ }^{13} \mathrm{C}$ NMR 和元素分析等手段进行了表征. 利用 UV/Vis 光谱 测定了化合物 $4 \mathrm{c}$ 和化合物 $4 \mathrm{e}$ 对不同金属离子的识别作
用，发现化合物 $4 \mathrm{c}$ 对 $\mathrm{Al}^{3+}$ 和 $\mathrm{Sn}^{2+}$ 有较好的识别效果，而 化合物 $4 \mathrm{e}$ 对 $\mathrm{Al}^{3+}$ 具有专一识别作用, 其与 $\mathrm{Al}^{3+}$ 络合的 物质的量比为 $1: 1$.

\section{1 结果与讨论}

\section{1 目标化合物的合成路线}

目标化合物的合成路线见 Scheme 1.

\section{2 化合物 4c 和 $4 \mathrm{e}$ 紫外性质测定}

以 $U V / V i s$ 光谱为检测手段, 初步研究了化合物 $\mathbf{4 c}$ 和 $4 \mathrm{e}$ 对金属离子的识别作用，结果发现 $4 \mathrm{c}$ 可以识别溶 液中的 $\mathrm{Al}^{3+}$ 和 $\mathrm{Sn}^{2+}$ (如图 1a 所示). 化合物 $4 \mathrm{c}$ 在 $420 \mathrm{~nm}$

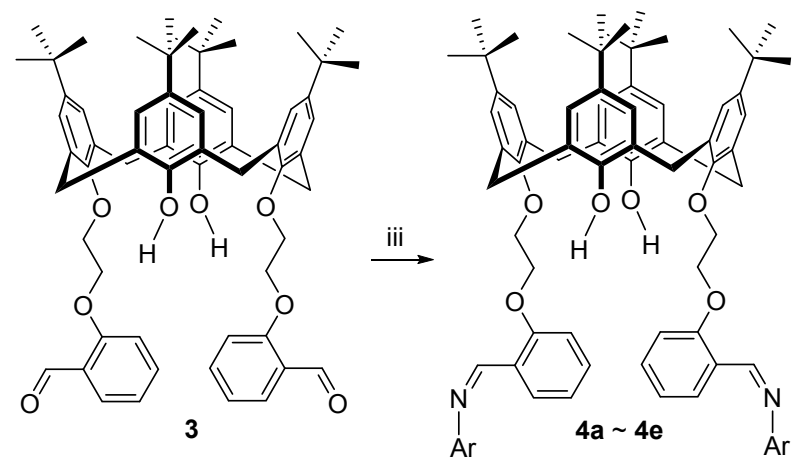

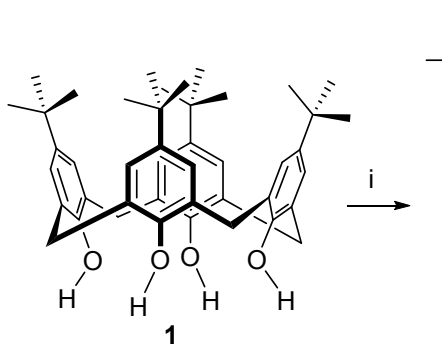

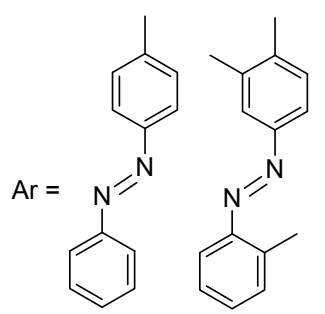

4a<smiles>Cc1ccc(/N=N/c2ccccc2)c2ccccc12</smiles>

4c

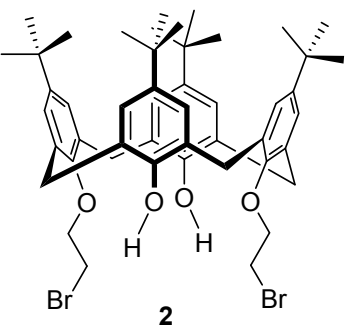<smiles>Cc1ccccc1N=Nc1ccc(C)c2ccccc12</smiles>

4d<smiles>Cc1ccc(/N=N/c2ccc(C)c3ccccc23)cc1</smiles>

$4 e$

Reagents and conditions: (i) 1,2-dibromoethane, $\mathrm{CH}_{3} \mathrm{CN}, \mathrm{K}_{2} \mathrm{CO}_{3}$, reflux, $18 \mathrm{~h}$; (ii) salicylaldehyde, $\mathrm{K}_{2} \mathrm{CO}_{3}$, reflux, $34 \mathrm{~h}$; (iii) 4-phenyl azoarylamine, methanol, reflux, $8 \mathrm{~h}$.

图式 1 目标化合物的合成

Scheme 1 Synthesis of the target compounds
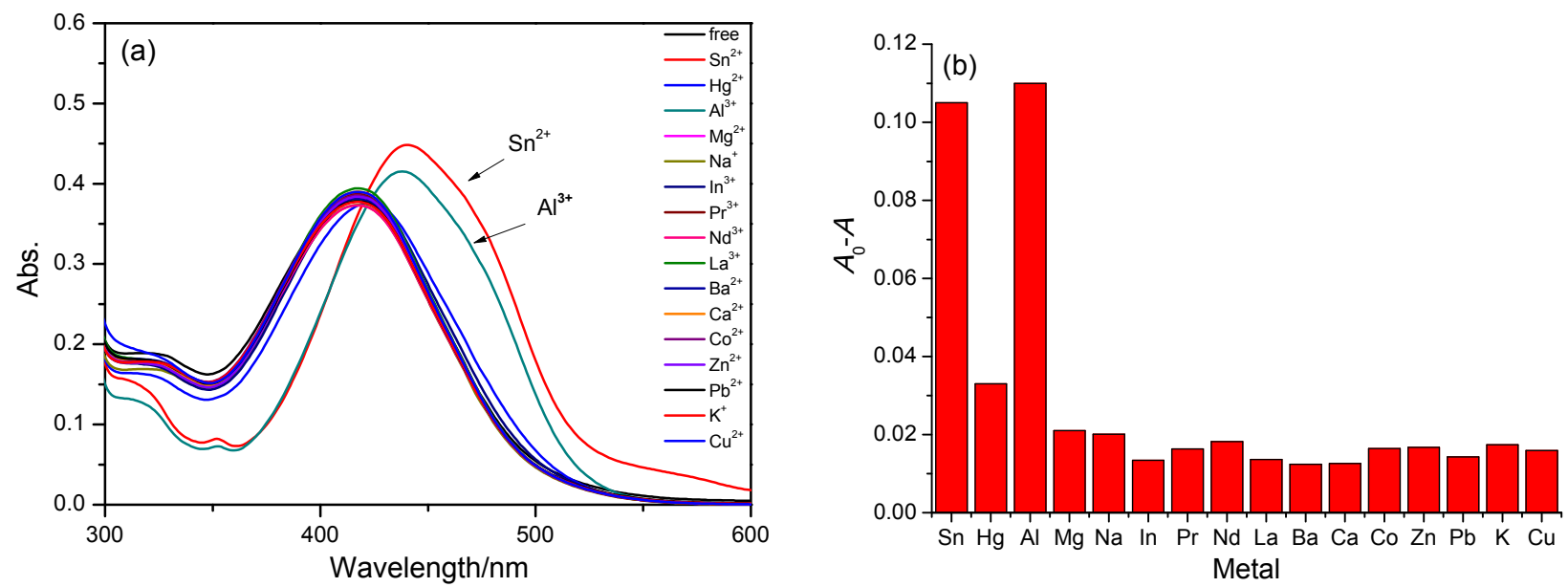

图 1 化合物 $4 \mathrm{c}$ 与不同金属离子作用的紫外光谱

Figure 1 UV/Vis spectra of compound $4 \mathbf{c}\left(1.0 \times 10^{-5} \mathrm{~mol} / \mathrm{L}\right)$ upon addition of various ions $\left(1.0 \times 10^{-5} \mathrm{~mol} / \mathrm{L}\right)$ in THF 
处出现强吸收峰, 在所有受试离子中只有 $\mathrm{Al}^{3+}$ 和 $\mathrm{Sn}^{2+}$ 可 使其吸收峰发生红移 $(\Delta \lambda=30 \mathrm{~nm})$. 图 $1 \mathrm{~b}$ 为柱状图, 其 中 $A_{0}$ 和 $A$ 分别为化合物 $4 \mathrm{c}$ 和 $4 \mathrm{c}$ 中加入不同的金属离 子后在 $350 \mathrm{~nm}$ 处的吸收波长.

实验还发现化合物 $4 \mathrm{e}$ 可以专一性地识别 $\mathrm{Al}^{3+}$ (如图 2a 所示). 化合物 $4 \mathrm{e}$ 在 $420 \mathrm{~nm}$ 处出现强吸收峰, 在所有 受试离子中, 只有加入 $\mathrm{Al}^{3+}$ 后其吸收峰发生了红移 $(\Delta \lambda$ $=15 \mathrm{~nm}$ ). 从柱状图(图 2b) 可以更明显看到化合物 $\mathbf{4 e}$ 对 $\mathrm{Al}^{3+}$ 的专一性识别作用. 通过物质的量比法测定化合物 $4 \mathrm{e}$ 与 $\mathrm{Al}^{3+}$ 络合的物质的量比为 $1: 1$ (如图 3 所示).
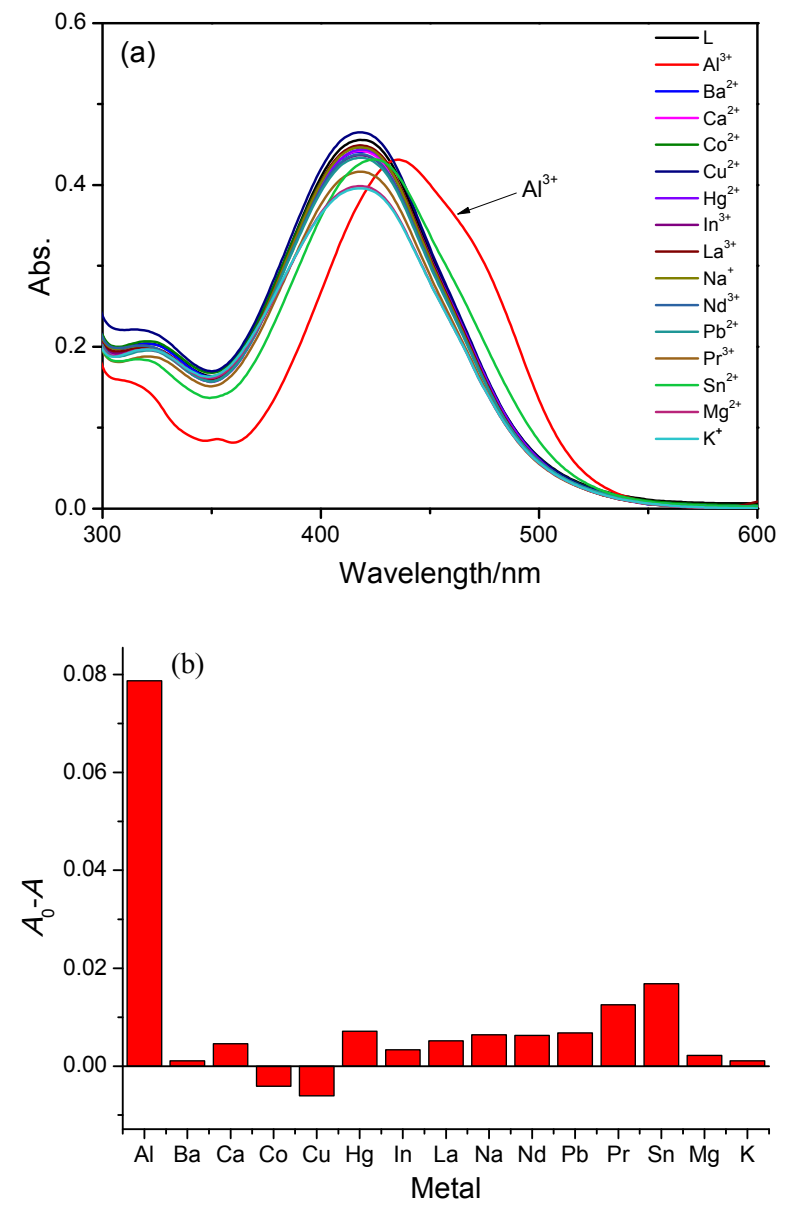

图 2 化合物 $4 \mathrm{e}\left(1.0 \times 10^{-5} \mathrm{~mol} / \mathrm{L}\right)$ 与不同金属离子 $\left(1.0 \times 10^{-5}\right.$ $\mathrm{mol} / \mathrm{L})$ 作用的紫外光谱

Figure 2 UV/Vis spectra of compound $4 \mathrm{e}\left(1.0 \times 10^{-5} \mathrm{~mol} / \mathrm{L}\right)$ upon addition of various ions $\left(1.0 \times 10^{-5} \mathrm{~mol} / \mathrm{L}\right)$ in THF

溶液的 $\mathrm{pH}$ 对于 $\mathrm{Al}^{3+}$ 的检测有着重要影响, 因此测 定了不同 $\mathrm{pH}$ 条件对化合物 $4 \mathrm{e}$ 与 $\mathrm{Al}^{3+}$ 络合体系紫外光谱 的影响(图 4). 图 4a 显示当溶液 $\mathrm{pH}=3 \sim 10$ 的变化时, 体系的颜色会从深黄色向亮黄色逐渐过渡, 并且在 $\mathrm{pH}$ 介于 5 8 之间时, 颜色基本保持不变. 图 $4 \mathrm{~b}$ 为不同 $\mathrm{pH}$ 条件下该络合体系在 $435 \mathrm{~nm}$ 处的紫外吸收强度, 从图 中可以清楚地看到当 $\mathrm{pH}$ 介于 $5 \sim 8$ 之间时, 该吸收峰强 度趋于稳定. 因此, 化合物 $4 \mathrm{e}$ 对 $\mathrm{Al}^{3+}$ 的检测最佳 $\mathrm{pH}$ 范

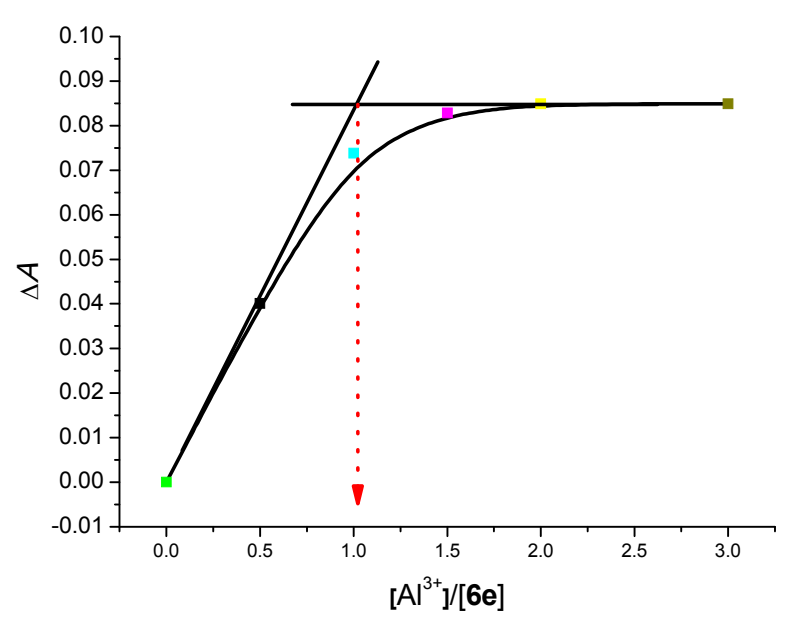

图 3 化合物 $4 \mathrm{e}$ 与 $\mathrm{Al}^{3+}$ 的物质的量比

Figure 3 The molar ratio of compound $4 \mathbf{e}$ with $\mathrm{Al}^{3+}$

围为 $5 \sim 8$ 之间. 本实验选择 $\mathrm{THF}$ 为溶剂时, 测定体系 的 $\mathrm{pH}=7.1$.
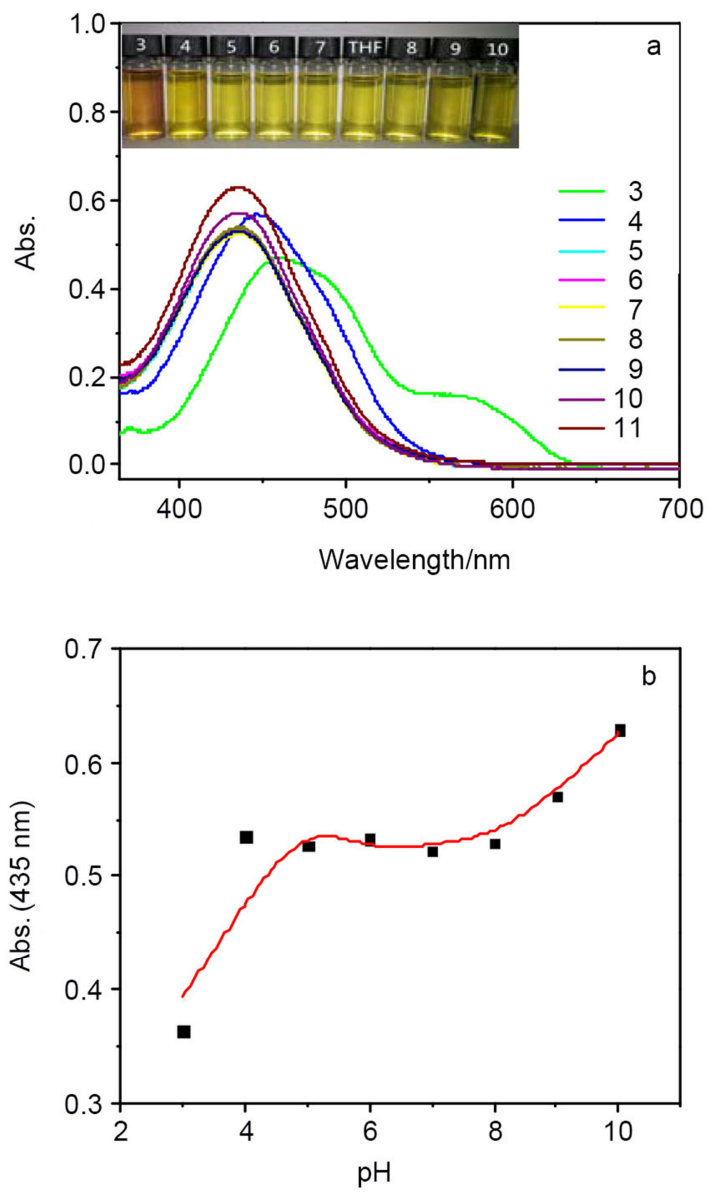

图 4 化合物 $4 \mathrm{e}\left(1.0 \times 10^{-5} \mathrm{~mol} / \mathrm{L}\right)$ 与 $\mathrm{Al}^{3+}\left(1.0 \times 10^{-5} \mathrm{~mol} / \mathrm{L}\right)$ 在不同 $\mathrm{pH}$ 下的紫外光谱

Figure $4 \mathrm{UV} / \mathrm{Vis}$ spectra of compound $4 \mathrm{e}\left(1.0 \times 10^{-5} \mathrm{~mol} / \mathrm{L}\right)$ and $\mathrm{Al}^{3+}\left(1.0 \times 10^{-5} \mathrm{~mol} / \mathrm{L}\right)$ under different $\mathrm{pH}$

亚氨基团中的 $\mathrm{N}$ 原子易于与金属离子发生络合作 
用, 其与金属离子配位后会使 $\mathrm{C}=\mathrm{N}$ 异构化受阻, 形成 刚性结构, 达到对金属离子检测的目的 ${ }^{[19,20]}$. 而偶氮杯 芳烃可以通过质子迁移形成互变异构改变其几何构型, 增加杂原子与金属离子配位的位点与几率 ${ }^{[21,22]}$. 由亚氨 和偶氮基团结合于杯芳烃下缘形成的下垂鳌合臂，与杯 芳烃特有的空腔及下缘原有的羟基相结合, 可以大大增 加该类化合物对金属离子的选择识别的能力. 主体分子 对 $\mathrm{Al}^{3+}$ 的选择性识别能力与其对 $\mathrm{Al}^{3+}$ 具有适当的几何 构型、合适的结合半径以及足够强的配位能力有关 ${ }^{[23]}$. 已有研究表明 $\mathrm{Al}^{3+}$ 易于同 $\mathrm{O} 、 \mathrm{~N}$ 等杂原子结合 ${ }^{[18,24]}$. 而 偶氮和亚氨基团作为下垂螯合臂的杯[4]芳烃化合物不 仅可以提供与 $\mathrm{Al}^{3+}$ 有效配位的多个杂原子, 而且其下垂 螯合臂能够通过互变异构达到与 $\mathrm{Al}^{3+}$ 配位所需要的几 何构型. 化合物 $4 \mathrm{c}$ 和 $4 \mathrm{e}$ 具有相似的结构, 唯一的区别 在于化合物 $4 \mathrm{e}$ 下缘的苯环上存在甲基取代基, 甲基的 空间位阻使得较大的原子或基团不容易进入到由两个 下垂螯合臂围成的空腔. $\mathrm{Sn}^{2+}$ 半径为 $0.69 \AA$, 而 $\mathrm{Al}^{3+}$ 的 半径为 $0.535 \AA$. 相对于 $\mathrm{Sn}^{2+}, \mathrm{Al}^{3+}$ 更容易进入到化合物 $4 \mathrm{e}$ 的空腔中发生络合作用.

\section{2 结论}

通过缩合和取代反应对杯芳烃的下缘进行修饰, 合 成了五个下缘同时含有偶氮和亚氨基团的新型杯 [4]芳 烃衍生物, 其结构通过 IR、 ${ }^{1} \mathrm{H} N \mathrm{NMR} 、{ }^{13} \mathrm{C} N \mathrm{NMR}$ 和元素 分析等手段进行了表征. 利用 UV/Vis 光谱分别测定了 化合物 $4 \mathrm{c}$ 和 $4 \mathrm{e}$ 对金属离子的识别作用, 结果显示化合 物 $4 \mathbf{c}$ 对溶液中 $\mathrm{Al}^{3+}$ 和 $\mathrm{Sn}^{2+}$ 具有较好的识别能力, 而化 合物 $4 \mathrm{e}$ 则表现出了对 $\mathrm{Al}^{3+}$ 的专一识别作用, 化合物 $4 \mathrm{e}$ 是一种潜在的检测 $\mathrm{Al}^{3+}$ 的探针化合物.

\section{3 实验部分}

\section{1 仪器与试剂}

X-4 型显微熔点测定仪; Spectrum One 型红外光谱 仪; Lamda25 UV/Vis 型紫外光谱仪( $\mathrm{KBr}$ 压片); Mercury-Vx-300 型超导核磁共振仪(TMS 为内标, $\mathrm{CDCl}_{3}$ 为溶 剂); FLASH EA1112 型元素分析仪. 化合物 $\mathbf{1}$ 和 2 分 别按照文献方法进行合成 ${ }^{[25,26]}$. 4-[(2-甲苯基)偶氮基]-1菜胺、4-[(4-甲苯基)偶氮基]-1-菜胺参考文献[27]的方法 进行合成. 其他试剂和溶剂均为市售分析纯或化学纯.

\section{2 化合物 $4 \mathrm{c}$ 和 $4 \mathrm{e}$ 的紫外光谱测定}

\subsection{1 不同金属离子的紫外光谱}

配制浓度为 $1.0 \times 10^{-3} \mathrm{~mol} / \mathrm{L}$ 的 $\mathrm{K}^{+} 、 \mathrm{Na}^{+} 、 \mathrm{Cu}^{2+}$ 、 $\mathrm{Ca}^{2+} 、 \mathrm{Pr}^{3+} 、 \mathrm{Ba}^{2+} 、 \mathrm{Co}^{2+} 、 \mathrm{In}^{3+} 、 \mathrm{~Pb}^{2+} 、 \mathrm{Zn}^{2+} 、 \mathrm{Nd}^{3+} 、$ $\mathrm{Hg}^{2+} 、 \mathrm{La}^{3+} 、 \mathrm{Al}^{3+} 、 \mathrm{Sn}^{2+}$ 的金属硝酸盐溶液和浓度为 $1.0 \times 10^{-3} \mathrm{~mol} / \mathrm{L}$ 的化合物 $4 \mathbf{c}$ 和 $4 \mathbf{e}$ 的 DMF 标准液作为
储备液.

准确量取 $0.25 \mathrm{~mL}$ 上述配制好的金属盐溶液分别加 入到 $25 \mathrm{~mL}$ 容量瓶中, 再准确量取 $0.25 \mathrm{~mL}$ 化合物 $\mathbf{4 c}$ 或 $4 \mathrm{e}$ 的标准液加入到上述各容量瓶中, 用 THF 定容至 刻度线, 震荡 $15 \mathrm{~min}$, 在紫外-可见分光光度计上, 以 $\mathrm{THF}$ 为参比液, 测定待测液的最大吸收波长.

3.2.2 化合物 $4 \mathrm{e}$ 与 $\mathrm{Al}^{3+}$ 在不同 $\mathrm{pH}$ 条件下的紫外光 谱

依次准确量取 $0.25 \mathrm{~mL}$ 化合物 $\mathbf{4 e}$ 的标准液加入到 9 个已编号的 $25 \mathrm{~mL}$ 容量瓶中, 再向各容量瓶中加入 0.25 $\mathrm{mL}$ 配制好的 $\mathrm{Al}^{3+}$ 溶液，调节各容量瓶 $\mathrm{pH}$ 依次为 $3,4,5$, 6,7, THF, 8,9 和 10 , 震荡 $15 \mathrm{~min}$, 在紫外-可见分光光 度计上, 以 $\mathrm{THF}$ 为参比液, 测定待测液的最大吸收波 长.

\section{3 合成与表征}

\subsection{1 化合物 $\mathbf{3}$ 的合成}

将化合物 $2(0.431 \mathrm{~g}, 0.50 \mathrm{mmol})$ 、水杨醛 $(0.183 \mathrm{~g}$, $1.50 \mathrm{mmol})$ 、无水碳酸钾 $(0.138 \mathrm{~g}, 1.00 \mathrm{mmol})$ 、乙腈 $40 \mathrm{ml}$ 依次加入到 $100 \mathrm{~mL}$ 圆底烧瓶中, 回流温度下反应. TLC 跟踪反应进程, $34 \mathrm{~h}$ 后停止反应. 抽滤, 蒸出溶剂, 残渣 用 $20 \mathrm{~mL}$ 二氯甲烷溶解, 每次用 $20 \mathrm{~mL}$ 蒸馏水水洗 2 次, 无水硫酸钠干燥过夜. 滤出无机盐, 粗产品用 $\mathrm{CH}_{2} \mathrm{Cl}_{2} / \mathrm{CH}_{3} \mathrm{CH}_{2} \mathrm{OH}$ 重结晶后经薄层色谱分离[展开剂 $V$ (乙酸乙酯) $: V($ 石油醚 $)=5: 2]$, 得淡绿色块状晶体 0.275 g, 产率 58.5\%. m.p. $211 \sim 212{ }^{\circ} \mathrm{C} ;{ }^{1} \mathrm{H}$ NMR $\left(\mathrm{CDCl}_{3}, 300 \mathrm{MHz}\right) \delta: 10.50(\mathrm{~s}, 2 \mathrm{H}, \mathrm{HC}=\mathrm{O}), 7.83 \sim 7.85$ (m, 2H, ArH), 7.50 7.54 (m, 2H, ArH), $7.49(\mathrm{~s}, 2 \mathrm{H}$, ArOH), 7.02 (s, 4H, ArH), 6.99 7.04 (m, 4H, ArH), 6.88 (s, $4 \mathrm{H}, \mathrm{ArH}), 4.40 \sim 4.55\left(\mathrm{~m}, 8 \mathrm{H}, \mathrm{OCH}_{2} \mathrm{CH}_{2} \mathrm{O}\right), 4.30(\mathrm{~d}$, $\left.J=8.4 \mathrm{~Hz}, 4 \mathrm{H}, \operatorname{ArCH}_{2} \mathrm{Ar}\right), 3.31(\mathrm{~d}, J=10.2 \mathrm{~Hz}, 4 \mathrm{H}$, $\left.\mathrm{ArCH}_{2} \mathrm{Ar}\right), 1.25\left[\mathrm{~s}, 18 \mathrm{H}, \mathrm{C}\left(\mathrm{CH}_{3}\right)_{3}\right], 1.02\left[\mathrm{~s}, 18 \mathrm{H}, \mathrm{C}\left(\mathrm{CH}_{3}\right)_{3}\right]$.

\subsection{2 化合物 $\mathbf{4 a} \sim \mathbf{4 e}$ 的合成}

在 $50 \mathrm{~mL}$ 的圆底烧瓶中加入化合物 $30.095 \mathrm{~g}(0.10$ $\mathrm{mmol}) 、 4$-苯基偶氮芳胺 $(0.25 \mathrm{mmol}) 、$ 甲醇 $25 \mathrm{~mL}$, 磁力 摚拌下回流反应, 反应瓶内逐渐析出大量沉淀. TLC 跟 踪反应, $8 \mathrm{~h}$ 后停止反应, 抽滤, 沉淀物经 $\mathrm{CH}_{2} \mathrm{Cl}_{2}$ / $\mathrm{CH}_{3} \mathrm{CH}_{2} \mathrm{OH}$ 重结晶分离, 分别得固体 $\mathbf{4 a} \sim \mathbf{4 e}$.

25,27-二羟基-26,28-二 $\{2$-[2-( $N$-(4-苯偶氮基苯基) 亚氨基次甲基苯氧基]乙氧基 $\}$ 对叔丁基杯 [4]芳烃(4a): 黄色颗粒状固体, 产率 $62.7 \%$. m.p. $254 \sim 256{ }^{\circ} \mathrm{C} ;{ }^{1} \mathrm{H}$ NMR $\left(\mathrm{CDCl}_{3}, 300 \mathrm{MHz}\right) \delta$ : 9.25 (s, $\left.2 \mathrm{H}, \mathrm{ArOH}\right), 8.21$ (d, $J=7.5 \mathrm{~Hz}, 2 \mathrm{H}, \mathrm{HC}=\mathrm{N}), 8.07$ (s, 2H, ArH), $7.90(\mathrm{~d}, J=8.1$ $\mathrm{Hz}, 4 \mathrm{H}, \mathrm{ArH}), 7.72(\mathrm{~d}, J=8.1 \mathrm{~Hz}, 4 \mathrm{H}, \mathrm{ArH}), 7.54 \sim 7.43$ (m, 8H, ArH), 7.22 (d, $J=9.3 \mathrm{~Hz}, 4 \mathrm{H}, \mathrm{ArH}), 7.08$ (d, $J=$ $7.5 \mathrm{~Hz}, 2 \mathrm{H}, \mathrm{ArH}), 6.95$ (d, J=25.5 Hz, 8H, ArH), 6.78 (d, 
$J=8.1 \mathrm{~Hz}, 2 \mathrm{H}, \mathrm{ArH}), 4.30\left(\mathrm{~d}, J=9.6 \mathrm{~Hz}, 4 \mathrm{H}, \mathrm{ArCH}_{2} \mathrm{Ar}\right)$, $4.17 \sim 4.28\left(\mathrm{~m}, 8 \mathrm{H}, \mathrm{OCH}_{2} \mathrm{CH}_{2} \mathrm{O}\right), 3.28(\mathrm{~d}, J=15.3 \mathrm{~Hz}, 4 \mathrm{H}$, $\left.\mathrm{ArCH}_{2} \mathrm{Ar}\right), 1.25\left[\mathrm{~s}, 18 \mathrm{H}, \mathrm{C}\left(\mathrm{CH}_{3}\right)_{3}\right], 1.04$ [s, $\left.18 \mathrm{H}, \mathrm{C}\left(\mathrm{CH}_{3}\right)_{3}\right]$; ${ }^{13} \mathrm{C}$ NMR $\left(\mathrm{CDCl}_{3}, 75 \mathrm{MHz}\right) \delta$ : 190.04, 160.45, 158.46, $157.04,154.71,152.49,150.11,149.13,147.24,141.66$, $130.93,132.99,132.67,130.63,128.89,127.72,125.70$, $125.14,124.52,123.89,122.73,121.81,120.92,113.74$, 111.47, 73.76, 66.64, 33.79, 32.01, 31.14, 30.49; IR (KBr) $v: 3392,3044,2861,1615,1587,1263,1193 \mathrm{~cm}^{-1}$. Anal. calcd for $\mathrm{C}_{86} \mathrm{H}_{90} \mathrm{O}_{6} \mathrm{~N}_{6} \cdot 0.5 \mathrm{CH}_{3} \mathrm{CH}_{2} \mathrm{OH}$ : C $78.7641, \quad \mathrm{H}$ 7.0655, N 6.3314; found C 78.8053, H 6.9584, N 6.1257.

25,27-二羟基-26,28-二\{2-[2-( $N$-(4-(2-甲基苯偶氮 基)-2-甲基苯基)亚氨基次甲基)苯氧基]乙氧基\}对叔丁 基杯[4]芳烃(4b): 橘黄色颗粒状晶体固体, 产率 61.6\%. m.p. $154 \sim 156{ }^{\circ} \mathrm{C} ;{ }^{1} \mathrm{H} \mathrm{NMR}\left(\mathrm{CDCl}_{3}, 300 \mathrm{MHz}\right) \delta$ : 9.14 (s, $2 \mathrm{H}, \mathrm{Ar}-\mathrm{OH}), 8.28(\mathrm{~d}, J=7.2 \mathrm{~Hz}, 2 \mathrm{H}, \mathrm{HC}=\mathrm{N}), 8.02(\mathrm{~s}, 2 \mathrm{H}$, $\mathrm{ArH}), 7.78$ (s, 2H, ArH), 7.60 (d, J=7.5 Hz, 2H, ArH), $7.35 \sim 7.50(\mathrm{~m}, 4 \mathrm{H}, \mathrm{ArH}), 7.32(\mathrm{~s}, 4 \mathrm{H}, \mathrm{ArH}), 7.25(\mathrm{~s}, 2 \mathrm{H}$ ArH), 7.07 (t, 2H, ArH), 6.96 (s, 4H ArH), 6.83 6.90 (m, $8 \mathrm{H}, \mathrm{ArH}$ ), 4.24 (d, $J=12.6 \mathrm{~Hz}, 4 \mathrm{H}, \mathrm{ArCH}_{2} \mathrm{Ar}$ ), $4.13 \sim 4.27$ (m, $\left.8 \mathrm{H}, \mathrm{OCH}_{2} \mathrm{CH}_{2} \mathrm{O}\right), 3.24$ (d, $J=11.7 \mathrm{~Hz}, 4 \mathrm{H}, \mathrm{ArCH}_{2} \mathrm{Ar}$ ), $2.71\left(\mathrm{~s}, 6 \mathrm{H}, \mathrm{ArCH}_{3}\right), 2.35\left(\mathrm{~s}, 6 \mathrm{H}, \mathrm{ArCH}_{3}\right), 1.22[\mathrm{~s}, 18 \mathrm{H}$, $\left.\mathrm{C}\left(\mathrm{CH}_{3}\right)_{3}\right], 1.05\left[\mathrm{~s}, 18 \mathrm{H}, \mathrm{C}\left(\mathrm{CH}_{3}\right)_{3}\right] ;{ }^{13} \mathrm{C} \mathrm{NMR}\left(\mathrm{CDCl}_{3}, 75\right.$ MHz) $\delta: 189.96,160.62,158.32,156.14,153.99,150.75$, $150.56,150.09,149.19,147.20,141.55,137.57,135.66$, $133.02,132.62,131.04,130.31,128.00,127.64,126.24$, $125.64,125.10,124.73,123.54,122.84,120.89,118.72$, $115.33,114.10,112.19,111.42,73.51,67.26,66.54,57.93$, 33.92, 33.70, 31.54, 30.97, 17.98, 17.51; IR (KBr) v: 3340, $3042,2958,2856,1617,1591,1192 \mathrm{~cm}^{-1}$. Anal. calcd for $\mathrm{C}_{90} \mathrm{H}_{98} \mathrm{O}_{6} \mathrm{~N}_{6} \cdot \mathrm{CH}_{3} \mathrm{CH}_{2} \mathrm{OH}: \mathrm{C}$ 78.5991, H 7.3394, N 5.9779; found $\mathrm{C} 78.3725$, H 7.6315, N 5.9652.

25,27-二羟基-26,28-二\{2-[2-( N-(4-苯偶氮基-1-䒬 基)亚氨基次甲基)苯氧基]乙氧基 $\}$ 对叔丁基杯 [4]芳烃 (4c): 红色颗粒状固体, 产率 55.6\%. m.p. 202 204 ${ }^{\circ} \mathrm{C}$; ${ }^{1} \mathrm{H} \mathrm{NMR}\left(\mathrm{CDCl}_{3}, 300 \mathrm{MHz}\right) \delta: 9.30$ (s, 2H, Ar-OH), 8.97 (d, $J=8.4 \mathrm{~Hz}, 2 \mathrm{H}, \mathrm{HC}=\mathrm{N}), 8.37 \sim 8.47(\mathrm{~m}, 4 \mathrm{H}, \operatorname{ArH})$, 8.01 (s, 6H, ArH), 7.68 (t, $J=15.3 \mathrm{~Hz}, 2 \mathrm{H}, \mathrm{ArH}), 7.58 \sim$ 7.69 (m, 10H, ArH), $7.36(\mathrm{t}, J=6.9 \mathrm{~Hz}, 2 \mathrm{H}, \mathrm{ArH}), 7.09(\mathrm{t}$, $J=7.5 \mathrm{~Hz}, 2 \mathrm{H}, \mathrm{ArH}), 6.92$ (s, $4 \mathrm{H}, \operatorname{ArH}), 6.84 \sim 6.88(\mathrm{~m}$, $10 \mathrm{H}, \mathrm{ArH}), 6.75$ (d, $J=8.1 \mathrm{~Hz}, 2 \mathrm{H}, \mathrm{ArH}), 4.17$ (d, $J=12.6$ $\left.\mathrm{Hz}, 4 \mathrm{H}, \mathrm{ArCH}_{2} \mathrm{Ar}\right), 4.09$ (d, $J=6.6 \mathrm{~Hz}, 8 \mathrm{H}, \mathrm{OCH}_{2} \mathrm{CH}_{2} \mathrm{O}$ ), $3.17\left(\mathrm{~d}, J=12.9 \mathrm{~Hz}, 4 \mathrm{H}, \mathrm{ArCH}_{2} \mathrm{Ar}\right), 1.20[\mathrm{~s}, 18 \mathrm{H}$, $\left.\mathrm{C}\left(\mathrm{CH}_{3}\right)_{3}\right], 1.01\left[\mathrm{~s}, 18 \mathrm{H}, \mathrm{C}\left(\mathrm{CH}_{3}\right)_{3}\right] ;{ }^{13} \mathrm{C}$ NMR $\left(\mathrm{CDCl}_{3}, 75\right.$ MHz) $\delta: 190.09,160.61,158.58,156.86,153.20,152.42$,
$150.05,149.12,147.23,145.21,141.54,133.08,131.91$, $130.53,129.25,128.97,126.00,127.63,127.08,125.94$, $125.61,125.07,124.12,122.94,113.47,113.01,111.53$, 73.49, 66.59, 33.93, 33.73, 31.81, 31.62, 30.99; IR (KBr) $v: 3385,3044,2956,2861,1615,1596,1188 \mathrm{~cm}^{-1}$. Anal. calcd for $\mathrm{C}_{90} \mathrm{H}_{98} \mathrm{O}_{6} \mathrm{~N}_{6} \cdot 0.5 \mathrm{CH}_{2} \mathrm{Cl}_{2}: \mathrm{C} 78.4744, \mathrm{H} 6.6204, \mathrm{~N}$ 5.8105; found C 78.220, H6.3098, N 5.5670.

25,27-二羟基-26,28-二\{2-[2-( $N$-(4-(2-甲基苯偶氮 基)-1-荎基)亚氨基次甲基)苯氧基]乙氧基 $\}$ 对叔丁基杯 [4]芳烃(4d): 红色颗粒状固体，产率 55.9\%. m.p. 184 $186{ }^{\circ} \mathrm{C} ;{ }^{1} \mathrm{H}$ NMR $\left(\mathrm{CDCl}_{3}, 300 \mathrm{MHz}\right) \delta: 9.27(\mathrm{~s}, 2 \mathrm{H}$, Ar-OH), $8.99(\mathrm{~d}, J=8.1 \mathrm{~Hz}, 2 \mathrm{H}, \mathrm{HC}=\mathrm{N}), 8.37 \sim 8.50(\mathrm{~m}$, $4 \mathrm{H}, \mathrm{ArH}), 7.97$ (s, 2H, ArH), 7.82 (d, J=7.5 Hz, 2H, ArH), $7.59 \sim 7.70(\mathrm{~m}, 8 \mathrm{H}, \mathrm{ArH}), 7.38(\mathrm{~d}, J=3.6 \mathrm{~Hz}, 6 \mathrm{H}, \mathrm{ArH})$, $7.10(\mathrm{t}, J=15 \mathrm{~Hz}, 2 \mathrm{H}, \mathrm{ArH}), 6.84 \sim 7.00(\mathrm{~m}, 10 \mathrm{H}, \mathrm{ArH})$, $6.75(\mathrm{~d}, J=8.7 \mathrm{~Hz}, 2 \mathrm{H}, \operatorname{ArH}), 4.2(\mathrm{~d}, J=12.6 \mathrm{~Hz}, 4 \mathrm{H}$, $\left.\mathrm{ArCH}_{2} \mathrm{Ar}\right), 4.05 \sim 4.22\left(\mathrm{~m}, 8 \mathrm{H}, \mathrm{OCH}_{2} \mathrm{CH}_{2} \mathrm{O}\right), 3.16(\mathrm{~d}, J=$ $\left.12.3 \mathrm{~Hz}, 4 \mathrm{H}, \mathrm{ArCH}_{2} \mathrm{Ar}\right), 2.76\left(\mathrm{~s}, 6 \mathrm{H}, \mathrm{ArCH}_{3}\right), 1.15$ [s, $\left.18 \mathrm{H}, \mathrm{C}\left(\mathrm{CH}_{3}\right)_{3}\right], 1.01\left[\mathrm{~s}, 18 \mathrm{H}, \mathrm{C}\left(\mathrm{CH}_{3}\right)_{3}\right] ;{ }^{13} \mathrm{C} \mathrm{NMR}\left(\mathrm{CDCl}_{3}\right.$, $75 \mathrm{MHz}) \delta$ : 190.13, 160.75, 158.59, 157.08, 152.45, $151.47,150.16,149.42,149.29,147.22,145.74,141.59$, $137.86,135.70,133.13,132.79,131.86,131.24,130.52$, $129.33,127.65,126.01,125.70,125.13,125.01,124.18$, $123.38,121.02,116.03,113.55,113.40,112.24,111.60$, $109.05,73.49,66.60,58.33,53.23,33.71,31.99,31.59$, 31.07, 18.26 17.81; IR (KBr) v: 3307, 3045, 2955, 2865, 1614, 1597, $1193 \mathrm{~cm}^{-1}$. Anal. calcd for $\mathrm{C}_{90} \mathrm{H}_{98} \mathrm{O}_{6} \mathrm{~N}_{6} \cdot$ $\mathrm{CH}_{3} \mathrm{CH}_{2} \mathrm{OH}$ : C 79.6429, H 7.0928, N 5.6864; found $\mathrm{C}$ 79.5432, H 7.3371, N 5.6227.

25,27-二羟基-26,28-二\{2-[2-( $N$-(4-(4-甲基苯偶氮 基)-1-荎基)亚氨基次甲基)苯氧基]乙氧基 $\}$ 对叔丁基杯 [4]芳烃(4e): 深色颗粒状固体, 产率 57.3\%. m.p. 238 $239{ }^{\circ} \mathrm{C} ;{ }^{1} \mathrm{H}$ NMR $\left(\mathrm{CDCl}_{3}, 300 \mathrm{MHz}\right) \delta: 9.29(\mathrm{~s}, 2 \mathrm{H}$, Ar-OH), $8.96(\mathrm{~d}, J=8.4 \mathrm{~Hz}, 2 \mathrm{H}, \mathrm{HC}=\mathrm{N}), 8.36 \sim 8.46(\mathrm{~m}$, 4H, ArH), 7.99 (s, 2H, ArH), 7.93 (d, $J=9 \mathrm{~Hz}, 4 \mathrm{H}, \mathrm{ArH})$, 7.65 (t, $J=15 \mathrm{~Hz}, 2 \mathrm{H}, \mathrm{ArH}), 7.57$ (d, $J=6.9 \mathrm{~Hz}, 2 \mathrm{H}, \mathrm{ArH})$, $7.50(\mathrm{~d}, J=7.8 \mathrm{~Hz}, 2 \mathrm{H}, \operatorname{ArH}), 7.36(\mathrm{t}, J=15.9 \mathrm{~Hz}, 6 \mathrm{H}$, ArH), 7.08 (t, $J=15 \mathrm{~Hz}, 2 \mathrm{H}, \mathrm{ArH}), 6.91(\mathrm{~s}, 4 \mathrm{H}, \mathrm{ArH}), 6.85$ (t, $J=12.6 \mathrm{~Hz}, 6 \mathrm{H}, \mathrm{ArH}), 6.74(\mathrm{~d}, J=8.4 \mathrm{~Hz}, 2 \mathrm{H}, \mathrm{ArH})$, 4.16 (d, $J=13.2 \mathrm{~Hz}, 4 \mathrm{H}, \mathrm{ArCH}_{2} \mathrm{Ar}$ ), 4.10 (t, 8H, $J=8.4$ $\mathrm{Hz}, \mathrm{OCH}_{2} \mathrm{CH}_{2} \mathrm{O}$ ), 3.16 (d, 4H, J=12.9 Hz, ArCH${ }_{2} \mathrm{Ar}$ ), $2.42\left(\mathrm{~s}, 6 \mathrm{H}, \mathrm{ArCH}_{3}\right), 1.20\left[\mathrm{~s}, 18 \mathrm{H}, \mathrm{C}\left(\mathrm{CH}_{3}\right)_{3}\right], 1.02[\mathrm{~s}, 18 \mathrm{H}$, $\left.\mathrm{C}\left(\mathrm{CH}_{3}\right)_{3}\right] ;{ }^{13} \mathrm{C} \mathrm{NMR}\left(\mathrm{CDCl}_{3}, 75 \mathrm{MHz}\right) \delta: 190.07,160.64$, $158.54,156.80,151.40,149.19,145.31,140.00,132.66$, $129.36,126.98,125.12,123.27,120.92,113.49,108.95$, 
73.51, 66.60, 52.94, 33.94, 33.76, 31.67, 31.01, 21.26; IR (KBr) v: 3365, 3035, 2953, 2870, 1616, 1600, $1616 \mathrm{~cm}^{-1}$. Anal. calcd for $\mathrm{C}_{90} \mathrm{H}_{98} \mathrm{O}_{6} \mathrm{~N}_{6} \cdot \mathrm{CH}_{3} \mathrm{CH}_{2} \mathrm{OH}$ : C 79.6429, $\mathrm{H}$ 7.0928, N 5.6864; found C 79.5373, H 7.2289, N 5.6169.

辅助材料(Supporting Information) 目标化合物核磁 共振氢谱和核磁共振碳谱数据. 这些材料可以免费从本 刊网站(http://sioc-journal.cn/)上下载.

\section{References}

[1] Xue, Y.; Yang, Y,; Gui, X. D.; Zhang, Z. B.; Huang, F. H. Acc. Chem. Res. 2012, 45, 1294.

[2] Fan, P.; Wan, L.; Shang, Y. S.; Wang, J.; Liu, Y. L.; Sun, X. Y.; Chen, C. Bioorg. Chem. 2015, 58, 88.

[3] Haluk, B.; Erdal, K.; Erhan, Z.; Ahmet, C. Electrochim. Acta 2011, 56, 2057.

[4] Chen, H. Q.; Fan, J. Z.; Hu, X. S.; Ma, J. W.; Wang, S. L.; Li, J.; Yu, Y. H.; Jia, X. S.; Li, C. J. Chem. Sci.. 2014, 50, 12420.

[5] Li, L.; Qi, W. G.; Wang, C.; Yan, C. G. Chin. J. Org. Chem. 2013, 33, 1804 (in Chinese). (李亮, 戚伟光, 王超, 颜朝国, 有机化学, 2013, 33, 1804.)

[6] Li, C. J. Chem. Commun. 2014, 50, 12420.

[7] Nag, J. K.; Pal, S.; Sinha, C. Trans. Met. Chem. 2001, 30, 523.

[8] Izatt, R. M.; Bradshaw, J. S.; Nielsen, S. A. Chem. Rev. 1985, 85, 271.

[9] Yang, Z. J.; Yin, C.; Zhang, L. P.; Zhong, Y.; Xu, H.; Mao, Z. P. Chin. J. Org. Chem. 2013, 33, 2607 (in Chinese).

(杨梓君, 尹冲, 张琳萍, 钟毅, 徐红, 毛志平, 有机化学, 2013, 33, 2607.)

[10] Diamond, D.; Nolan, K. Anal. Chem. 2001, 73, 22.

[11] Forster, R. J.; Cadogan, A.; Diaz, M. T. Sens. Actuators, B 1991, 4,
325.

[12] Kumar, M.; Babu, J. N.; Bhalla, V; Dhir, A. Inorg. Chem. Commun. 2009, 12, 332.

[13] Vogtle, F. Pure Appl. Chem. 1980, 52, 2405.

[14] Haluk, B.; Erdal, K.; Erhan, Z.; Ahmet, C. Electrochim. Acta 2011, 56, 2017.

[15] Sahana, A.; Banerjee, A.; Das, S.; Lohar, S.; Karak, D.; Sarkar, B.; Mukhopadhyay, S. K.; Mukherjee, A. K.; Das, D. A. Org. Biomol. Chem. 2011, 9. 5523.

[16] Ren, J.; Tian, H. Sensors 2007, 12, 3166.

[17] Nayak, P. Environ. Res. 2002, 89, 101.

[18] Ma, T.; Zhang, J.; Liu, L. Z.; He, Y.; Zhang, Z. T. Chin. J. Org. Chem. 2014, 34, 1780 (in Chinese). (马拓, 张瑾, 刘龙珠, 贺云, 张尊听, 有机化学, 2014, 34, 1780.)

[19] Wang, D. F.; Ke, Y. C.; Guo, H. X.; Chen, J. H.; Weng, W. Spectrochim. Acta, Part A 2014, 122, 268.

[20] Gupta, V. K.; Singh, A. K.; Kumawat, L. K. Sens. Actuators, B: 2014, 195, 98.

[21] Fan, P.; Jin, Z.; Pan, Y., Liu, G. J. Chem. J. Chin. Univ. 2009, 30, 724 (in Chinese) (范平，金轩，潘懿，刘光锦，高等学校化学学报, 2009, 30, 724.)

[22] Bonvallet, P. A.; Mullen, M. R.; Evans, P. J.; Stoltz, K. L.; Story, E. N. Tetrahedron Lett. 2011, 52, 1117.

[23] Liu, Z. D.; Xu, H. J.; Sheng, L. Q. Chin. J. Spectrosc. Lab. 2011, 28, 393 (in Chinese). (刘昭第, 徐华杰, 盛良全, 光谱实验室, 2011, 28, 393.)

[24] Dhara, A.; Jana, A.; Guchhait, N.; Kar, S, K. J. Luminesc. 2014, $369,375$.

[25] David, G.; Muzafer, L. D. J. Org. Chem. 1986, 51, 742.

[26] Zhao, B. T.; Wang, L.; Ye, B. X. Chin. J. Org. Chem. 2006, 26, 1562 (in Chinese). (赵邦屯，王璐，冶保献，有机化学, 2006, 26, 1562.)

[27] Sekhr, L.; Bebba, A. A.; Hassini, Z. Asian J. Chem. 2005, 17, 2455.

(Li, L.; Fan, Y.) 